\title{
Les élections européennes de 2014 : une analyse comparative des résultats aux échelles nationale et régionale
}

\section{Christian Vandermotten and Pablo Medina Lockhart}

\section{(2) OpenEdition}

1 Journals

\section{Electronic version}

URL: http://journals.openedition.org/echogeo/14303

DOI: 10.4000/echogeo.14303

ISSN: 1963-1197

\section{Publisher}

Pôle de recherche pour l'organisation et la diffusion de l'information géographique (CNRS UMR 8586)

\section{Electronic reference}

Christian Vandermotten and Pablo Medina Lockhart, « Les élections européennes de 2014 : une analyse comparative des résultats aux échelles nationale et régionale », EchoGéo [Online], Sur le Vif, Online since 22 July 2015, connection on 01 May 2019. URL : http://journals.openedition.org/ echogeo/14303; DOI : 10.4000/echogeo.14303

This text was automatically generated on 1 May 2019.

\section{c) (i)}

EchoGéo est mis à disposition selon les termes de la licence Creative Commons Attribution - Pas d'Utilisation Commerciale - Pas de Modification 4.0 International 


\title{
Les élections européennes de 2014 : une analyse comparative des résultats aux échelles nationale et régionale
}

\author{
Christian Vandermotten and Pablo Medina Lockhart
}

\section{Introduction}

1 Cet article n'a d'autre ambition qu'empirique, à savoir une présentation comparative, « sur le vif ", à l'échelle régionale, des résultats des élections européennes de mai 2014, ainsi que leur évolution par rapport aux élections précédentes de $2009^{1}$.

2 Les entités territoriales considérées pour la présentation des résultats correspondent aux niveaux NUTS 3 de la classification régionale européenne (départements français, provinces en Italie, en Espagne, aux Pays-Bas, etc.) ou, dans les pays où les unités NUTS 3 sont trop petites, NUTS 2, comme en Allemagne (Regierungsbezirke) ou en Belgique (provinces) ; le découpage a tenté de viser au mieux la cohérence des tailles et la prise en compte de la réalité des nuances politiques régionales, à une échelle compatible avec une analyse pan-européenne. La présentation des résultats fait appel à des anamorphoses cartographiques, avec des surfaces des entités régionales proportionnelles au nombre d'électeurs inscrits en 2014 : une telle représentation permet mieux de juger des résultats des entités métropolitaines, souvent négligées du fait de leur petite superficie sur des représentations classiques, et ramène à leur juste poids les entités rurales et périphériques peu peuplées. Le choix cartographique a été fait de privilégier la comparaison de la force respective des différentes familles politiques plutôt que la géographie interne de chacune d'elles. Il en résulte une discrétisation commune aux différentes cartes, et dès lors nécessairement « rudimentaire », avec des limites de classes « rondes », voire la non-utilisation de certaines classes pour des familles n'atteignant 
nulle part des scores élevés : c'est le prix à payer pour une parfaite comparabilité entre les cartes.

$3 \mathrm{Si}$, à première vue, les élections européennes, qui se déroulent au même moment dans les 28 pays membres de l'Union, peuvent paraitre un moment privilégié pour saisir l'ensemble du panorama électoral du continent, de grandes précautions doivent néanmoins être prises dans l'interprétation des résultats. Ainsi, même une analyse empirique impose quelques détours théoriques et méthodologiques, pour tenir compte de la complexité des comparaisons internationales en matière de géographie électorale et des précautions qu'elles imposent. Ces difficultés relèvent de plusieurs facteurs :

- La difficulté de classer de manière univoque et de regrouper les différents partis en lice, nonobstant leur appartenance à des familles politiques constituées au Parlement européen.

- Le positionnement différent des élections européennes dans les cycles et les conjonctures politiques des différents États.

- Des attitudes différenciées des diverses opinions publiques nationales envers la construction européenne, ce qui se traduit non seulement dans les choix partisans des électeurs, mais aussi par des niveaux de participation au vote extrêmement différents selon les pays, sans parler de ceux où le vote est théoriquement obligatoire. À l'inverse, les élections européennes se déroulant partout à la représentation proportionnelle par liste, soit au niveau national, soit à celui de grandes circonscriptions, elles sont moins influencées par le souci de voter utile ou par les stratégies des électeurs visant à bloquer l'élection d'un candidat qu'ils rejettent absolument que ce n'est le cas pour les élections nationales dans les pays à scrutin uninominal à un (la Grande-Bretagne) ou à deux tours (la France).

4 L'interprétation des résultats doit aussi prendre en compte le fait que les élections européennes sont des élections intermédiaires dans les calendriers politiques nationaux, pouvant dès lors d'autant plus donner lieu à des votes de protestation qu'elles apparaissent comme moins décisives aux yeux de beaucoup d'électeurs. Ce dont témoigne aussi un taux d'abstention bien plus élevé, qui sera analysé ci-après.

\section{La classification des partis au niveau européen}

Procéder à une analyse des résultats électoraux à l'échelle européenne impose évidemment de regrouper les résultats des différents partis en un petit nombre de catégories, un exercice nécessairement réducteur et bien plus complexe encore que s'il ne s'agissait que de situer les formations politiques dans leurs cadres nationaux (Vandermotten, 2011). Ceci sans même évoquer la difficulté de situer avec précision le programme et les positions politiques de candidats indépendants ou de certains très petits partis, voire d'identifier ce qui se cache derrière la catégorie "autres", dans laquelle sont regroupés par les statistiques électorales de certains pays les résultats confidentiels de petites listes, mais qui peuvent néanmoins, en les additionnant, représenter quelques pourcents des votants.

Plusieurs positions théoriques sont possibles pour procéder à ces regroupements.

7 Se reporter à la théorie des clivages, fondée par Rokkan, Lipset et Seiler (Lipset \& Rokkan (eds.), 1967 [trad. franç., 2008] ; Rokkan, 1970 ; Seiler, 2003). Ceci amènerait à isoler une famille catholique et une famille libérale sur l'axe fonctionnel du clivage Église-État issu de la révolution nationale (en tout cas dans les pays catholiques); une famille agrarienne sur le versant rural de l'axe territorial issu du clivage de la révolution industrielle ; une famille 
conservatrice, opposée aux familles socialiste et communiste sur l'axe fonctionnel possédants-travailleurs. Restent encore à situer les écologistes et la droite extrême et identitaire contemporaine, qui s'insèrent chacune mal dans cette théorie, sauf à considérer de nouveaux axes de clivage, comme économie-écologie, cosmopolitisme - identité, voire modernisme - post-modernisme. Cela pourrait mener à placer de ce (seul) dernier point de vue écologistes et droite extrême et identitaire du même côté de cet axe, un refus de la modernité économiste globalisée, opposés alors à l'ensemble des autres familles, qui toutes prônent le primat de la croissance, ne divergeant plutôt que sur les modalités de sa régulation et de la répartition de ses fruits. Mais écologie politique et droite extrême seraient alors opposées sur les clivages cosmopolitisme - identité/localisme et écologie - économie. Il faut reconnaitre à cette proposition un côté "bricolage», les clivages rokkaniens ne correspondant pas seulement à des simples oppositions, mais s'inscrivant dans les logiques historiques fondamentales des révolutions nationales et industrielles. Et enfin, que faire dans l'application de cette théorie de petits partis libertaires, comme les partis pirates, ou catégoriels, les plus délicats à positionner quelle que soit la base classificatoire retenue (des femmes aux pensionnés en passant par les automobilistes et les buveurs de bière)? Néanmoins, la principale limitation à une application pratique de la théorie des clivages tient à son caractère génétique, historique, plutôt qu'opérationnel. Elle permet difficilement de prendre en compte le fait que les partis contemporains se positionnent sur un ensemble d'enjeux sociétaux, et pas uniquement sur ceux qui ont éventuellement déterminé leur fondation ou qui prévalaient à cette époque. Ces évolutions rendent en particulier difficile l'usage de cette théorie pour éventuellement distinguer aujourd'hui partis chrétiens-démocrates, conservateurs, libéraux, agrariens, parfois renommés en modérés en liaison avec la très forte réduction de la population rurale agricole.

Se contenter, de manière purement pragmatique, de considérer l'appartenance des partis aux groupes politiques au Parlement européen, en la complétant éventuellement par leur appartenance à différentes internationales partisanes. Si cette solution peut paraître en première approche adéquate pour une analyse des résultats des élections européennes, elle ne va toutefois pas sans soulever de nombreuses difficultés et insuffisances :

- les groupes politiques peuvent être très hétérogènes quant aux positions des partis qu'ils rassemblent. Pensons par exemple, sous la précédente législature européenne, à la NVA flamande, alors membre du groupe Verts/Alliance libre européenne malgré des positions, tant économiques qu'écologiques, très à droite ; ou encore au groupe démocrate-chrétien et conservateur du PPE où se retrouvent le $\mathrm{cdH}$, les humanistes ex-démocrates-chrétiens belges francophones, programmatiquement plus à gauche que le Labour britannique, et les très droitières et conservatrices FIDESZ hongroise de Viktor Orban ou CSU bavaroise ;

- des partis peuvent changer de groupe politique d'une élection à l'autre (ainsi le Parti national libéral roumain, passé du groupe libéral ADLE au PPE; ou la NVA, déjà citée plus haut, passée du groupe Verts/Alliance libre européenne au groupe eurosceptique conservateur ECR, qui correspond effectivement bien mieux à ses positions politiques) ;

- reste encore le problème des non-inscrits, que l'on ne peut certes ramener à une catégorie résiduelle de "divers "; celui d'élus d'un même parti qui se séparent au moment de leurs choix d'inscription à un groupe; celui des petits partis non représentés au Parlement européen ; sans parler des comparaisons avec les pays non-membres de l'Union européenne.

Se fonder sur une analyse des positionnements programmatiques des partis (Trechsel \& Mair, 2009). Cet exercice peut être mené sur la base d'une analyse de correspondances fondée 
sur une lecture des programmes et sur les interviews de responsables des partis ou d'experts nationaux. Elle permet de dégager empiriquement deux axes fondamentaux, d'une part un axe de type gauche - droite classique, qui oppose les partis sur une base économique (plus de régulation, vs. plus de libéralisme), d'autre part un axe de type socio-culturel (plus de libéralisme sur le plan moral et culturel, plus de valeurs "cosmopolites», vs. plus de protection de l'ordre établi, de valeurs identitaires, de morale traditionnelle, etc.). Ce constat renvoie implicitement à la théorie du changement post-matérialiste de valeurs d'Ingelhart $(1977,2007)$, bien qu'il faille constater que le premier axe rend compte d'une part nettement plus importante de la variance totale des positions programmatiques que le deuxième. L'illustration 1 situe sur ces axes les ensembles regroupant les partis politiques appartenant aux différentes familles représentées au Parlement européen. Les intersections entre les différents ensembles sont larges. On remarquera aussi que cette analyse devrait en outre faire intervenir d'autres axes, indépendants des deux principaux représentés. On peut ainsi observer que, si les trois ensembles rassemblant les groupes Gauche unitaire européenne, Verts/ Alliance libre européenne et Socialistes et Démocrates se positionnent tous à gauche sur l'axe socio-économique, avec de larges recouvrements, cela ne rend pas compte d'une prise en compte beaucoup plus affirmée des questions écologiques par le groupe des Verts, quitte à y sacrifier la croissance dans ses formes traditionnelles, ni de positions nettement plus eurocritiques dans le groupe GUE. Mais ces différences de positionnement peuvent aussi traverser une même famille; ainsi, à l'intérieur de la Gauche unitaire européenne s'oppose par exemple un parti communiste historique, comme le PC tchèque, à des mouvements d'extrême-gauche issus du rassemblement d'anciens communistes critiques et d'écologistes. Un autre axe indépendant, qui concerne cette fois plutôt les partis de centre-droit, que l'on peut globalement considérer comme porteurs des valeurs des establishments politico-économiques ${ }^{2}$, est celui concernant l'attitude envers la construction européenne: si les groupes libéraux et populaires européens sont globalement favorables à l'approfondissement de la construction européenne (avec quelques exceptions notables, en particulier, au sein du PPE, le FIDESZ hongrois), il n'en va pas de même du groupe eurosceptique ECR, qui entend limiter au mieux la construction européenne à un vaste libre marché, sinon s'en retirer, même si c'est plus sur la base d'un ultra-libéralisme économique cosmopolite et atlantiste que d'un repli identitaire nationaliste, du moins en ce qui concerne les principaux partis de ce groupe, comme les conservateurs britanniques et l'ODS tchèque. 
Illustration 1 - Positionnement programmatique des familles politiques européennes

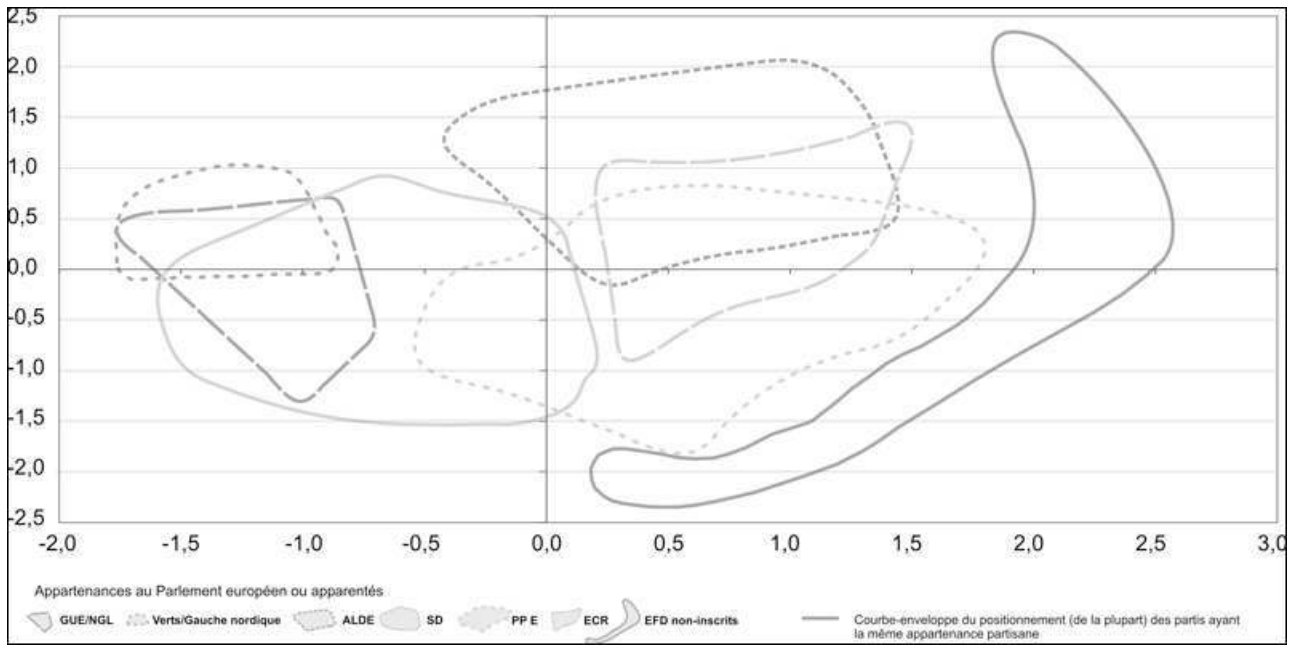

L'axe horizontal rend compte des positions sur le plan socio-économique ; l'axe vertical rend compte des positions sur le plan socio-culturel.

Source : C. Vandermotten, 2011, simplifié, d'après les données de A.H. Trechsel \& P. Mair,2009

Se fonder sur le positionnement des électeurs, tel qu'il est rendu par les Eurobaromètres (B rechon, 2002 ; Tilburg University \& Gesis Leibniz Institute For The Social Sciences, 1999 \& 2008). Ce positionnement permet lui aussi de situer la position moyenne des électeurs des partis soit sur un axe unique gauche - droite, soit dans une représentation sur deux ou plusieurs axes indépendants. Elle ne donne pas exactement les mêmes résultats qu'une analyse sur la base des programmes des partis : en général, les électeurs se positionnent sur des valeurs plus à droite que le contenu des programmes partisans, sauf les électeurs des partis d'extrême-droite, qui se positionnent plus à gauche.

Finalement, confrontés à l'ensemble de ces difficultés théoriques et pratiques, nous avons opté pour une solution très pragmatique, en acceptant par avance les diverses objections auxquelles elle pourra donner lieu. Il s'agit de limiter l'analyse à un petit nombre de grands ensembles, très englobants, fondés sur des critères simples, basés sur des enjeux socio-politiques majeurs, que nous allons décrire ci-dessous, sans toutefois toujours pouvoir entièrement éviter l'impact des regroupements en familles politiques au Parlement européen (par exemple, le FIDESZ hongrois pose ainsi un problème). Des regroupements larges atténuent en effet les difficultés de classement, même si elles peuvent subsister, par exemple pour les partis de type Verts-Gauche ou pour des cartels électoraux rassemblant extrême-gauche et écologistes, sur une base régionaliste, en Espagne, ou encore pour cette curieuse alliance entre sociaux-démocrates et le petit Parti conservateur en Roumanie. Les petits partis, catégoriels ou non, et les candidats indépendants ont été attribués aux différents ensembles soit sur la base d'une lecture de leur programme, soit en fonction de leurs alliances internationales éventuelles. Dans quelques cas, il n'est toutefois resté d'autre solution que de les laisser dans une catégorie résiduelle, qui ne sera pas étudiée.

12 Nous avons donc limité l'analyse des résultats aux catégories suivantes (tableau 1):

- Les partis développant une critique de gauche du modèle social, politique et économique dominant. Il s'agit d'une part des partis d'extrême-gauche issus des traditions politiques du mouvement communiste international, mais aussi de nouvelles expressions de contestations sociales 
(Syriza en Grèce, Podemos en Espagne), parfois même marquées de prises en compte radicales de revendications nationales (le Sinn Fein irlandais). Si cette critique de gauche reste très dominée par des positionnements contestataires sociaux et économiques classiques dans les partis qui viennent d'être évoqués, il faut d'autre part y ajouter les partis verts, où l'écologie et le post-modernisme économique représentent une base forte de la contestation politique, même si elle est loin d'être exclusive. Ces partis développent aussi en général une position critique plus ou moins radicale envers les formes prises par la construction européenne, tout en ne remettant pas fondamentalement en cause l'idée européenne elle-même, mais en voulant la redéployer sur d'autres fondements (unité des mouvements de travailleurs ou projet écologique commun). On peut ajouter à cette catégorie des petits partis dont la contestation, globalement plutôt de gauche, se positionne plus sur le plan socio-culturel, voire sur des bases assez individualistes ou libertaires : partis pirates, humanistes, féministes, etc. Nous avons donc cartographié l'extrême-gauche et les partis verts et assimilés à la fois ensemble (contestation de gauche du système établi) et séparément (pour bien mettre en évidence la contestation de type écologique et postmoderne).

- Les partis sociaux-démocrates. C'est le groupe globalement le plus homogène, même si cette homogénéité a quelque peu régressé depuis l'ouverture à l'Europe centre-orientale et s'il peut y avoir une large distance sur un axe gauche - droite, entre le PS belge francophone et le Labour britannique, dont le programme se situe plus à droite que celui de certains partis membres du PPE ou de l'ADLE (comme D'66 aux Pays-Bas). Ce groupe rassemble des partis qui sont ou sont potentiellement des partis de gouvernement, mais prônent le maintien d'une certaine régulation de l'économie et l'attention au partage des fruits de la richesse, tout en acceptant, avec des nuances, les contraintes imposées par les règles européennes, par exemple en matière de rigueur et d'austérité budgétaires.

- Le centre-droit et la droite d'establishment pro-européens. Nous nous sommes résolus à fusionner les partis qui relèvent au Parlement européen des groupes ADLE et PPE ou qui en sont proches. Cela crée certes un ensemble très vaste, mais ce regroupement rassemble les partis représentant les forces politiques proches de l'establishment et qui déterminent les règles de la construction européenne, même si c'est avec une gamme de nuances entre plus de fédéralisme ou une préservation résolue des souverainetés nationales. En collaboration avec les partis sociaux-démocrates, ce sont les partis de ce rassemblement composite qui fixent l'agenda politique européen et maîtrisent le fonctionnement des institutions. Distinguer les partis libéraux et conservateurs/démocrates-chrétiens n'eût pas toujours été aisé, ni opérationnel : certains partis ont migré sans aucun état d'âme d'un groupe politique à l'autre au Parlement européen; l'anticléricalisme des partis libéraux historiques appartient au passé, de même le plus souvent que la défense résolue des valeurs chrétiennes par les partis de tradition démocrate-chrétienne; même sur le plan de l'ouverture socioculturelle, bien des partis conservateurs ou démocrates-chrétiens n'ont plus rien à envier à certains partis de filiation libérale, même si dans l'ensemble ces derniers restent plus ouverts de ce point de vue. En fin de compte l'éventail des positions est tout aussi grand à l'intérieur des groupes PPE et ADLE qu'entre ces deux groupes.

- Le centre-droit et la droite d'establishment eurosceptique. En revanche, il est apparu nécessaire $\mathrm{d}$ 'isoler les partis qui, tout en étant des partis de gouvernement, défendent des positions très critiques et/ou d'opposition à l'égard de la construction européenne. Ces partis sont pour l'essentiel réunis dans le groupe $\mathrm{ECR}$, du moins pour ceux qui possèdent une représentation parlementaire. Les positions très nationalistes et identitaires du FIDESZ eussent pu le ramener dans ce groupe; nous l'avons finalement laissé dans le groupe précédent en 
fonction de son appartenance (quelque peu contestée en interne) au PPE, mais il faudra tenir compte de telles ambiguïtés dans les commentaires des cartes. Nous avons ajouté à ce groupe l'ensemble des partis de centre-droit et de droite d'establishment suisses et norvégiens adversaires de l'adhésion à l'Europe.

- La droite extrême identitaire. Les partis rassemblés ici ont certes des histoires et des positions très diverses, qui empêchent d'ailleurs la plupart d'entre eux de se rassembler en un groupe au Parlement européen et les renvoie donc parmi les non-inscrits, si l'on excepte les partis rassemblés dans le groupe ELDD (EFD) (Europe de la liberté et de la démocratie directe) sous la houlette de l'UKIP britannique. Ils peuvent être, parfois simultanément à travers diverses tendances internes, de tradition néo-fasciste ou néo-nazie, de droite nationaliste identitaire extrême, ultra-conservatrice, religieuse et moralisatrice, populiste, rassemblant les électeurs frustrés par les conséquences au quotidien de la crise économique et sociale et par leur perception, réelle ou fantasmée, des impacts de l'immigration. Parfois, ils défendent, sur une base réactionnaire, des intérêts catégoriels. On peut aussi y renvoyer des partis anarchistes anti-système aux idéologies populistes fumeuses (5 Stelle en Italie). Les partis de cette catégorie partagent un refus de la construction européenne sur des bases nationalistes, identitaires, s'opposant aux forces économiques dominantes de l'establishment ; ils peuvent aller jusqu'à une contestation du capitalisme, du moins du capitalisme globalisé dominant.

Un tableau, reporté en annexe, reprend la position dans cette classification de l'ensemble des partis ayant obtenu plus de 3,5\% des suffrages exprimées, au niveau national, soit en 2009, soit en 2014, à l'exclusion de quelques candidats indépendants.

\section{Des cartes qui restent très dominées par les traditions politiques nationales, pour des enjeux européens qui apparaissent lointains et peu concrets aux électeurs}

Les comportements des votants aux élections européennes restent largement déterminés par les enjeux politiques nationaux et s'inscrivent dans les systèmes partisans des différents pays : une analyse isolant la variance inter-États de la variance inter-régionale est à cet égard édifiante (tableau 1). Ses résultats seraient plus spectaculaires encore si les deux parties de l'Allemagne, dont les comportements politiques restent très tranchés, étaient considérés comme deux ensembles distincts.

Tableau 1- Analyse de variance des résultats des élections européennes

\begin{tabular}{|l|l|l|}
\hline & $\begin{array}{l}\text { Part de la variance inter- } \\
\text { États dans la variance totale } \\
\text { des résultats en 2014 }\end{array}$ & $\begin{array}{l}\text { Part de la variance inter-États } \\
\text { dans la variance totale des } \\
\text { évolutions 2009-2014 }\end{array}$ \\
\hline $\begin{array}{l}\text { Taux de participation (part des } \\
\text { votes valables dans le total des } \\
\text { inscrits) }\end{array}$ & $84,4 \%$ & $47,2 \%$ \\
\hline $\begin{array}{l}\text { Pourcentage obtenu par les } \\
\text { partis d'extrême-gauche }\end{array}$ & $79,1 \%$ & $87,8 \%$ \\
\hline
\end{tabular}




\begin{tabular}{|l|l|l|}
\hline $\begin{array}{l}\text { Pourcentage obtenu par les } \\
\text { partis écologistes }\end{array}$ & $54,5 \%$ & $84,3 \%$ \\
\hline $\begin{array}{l}\text { Pourcentage obtenu par les } \\
\text { partis sociaux-démocrates }\end{array}$ & $68,1 \%$ & $91,8 \%$ \\
\hline $\begin{array}{l}\text { Pourcentage obtenu par les } \\
\text { partis de centre-droit et de } \\
\text { droite eurosceptique }\end{array}$ & $84,3 \%$ & $85,7 \%$ \\
\hline $\begin{array}{l}\text { Pourcentage obtenu par les } \\
\text { partis d'extrême-droite et de } \\
\text { droite identitaire }\end{array}$ & $87,0 \%$ & $73,6 \%$ \\
\hline
\end{tabular}

Rappelons que la variance est la moyenne du carré des écarts d'une série statistique par rapport à sa moyenne. La variance totale des pourcentages obtenus par chaque famille politique dans chacune des 660 subdivisions régionales étudiées est la somme de la variance entre les moyennes (non pondérées) des 28 pays de l'échantillon (la variance inter-États) et son complément à la variance totale des 660 valeurs. Ce dernier poste représente donc la part de l'ensemble de la variance qui n'est pas « expliquée » par l'appartenance des entités régionales à leur état et à son comportement politique moyen. Imaginons que nous ayons un échantillon de 20 entités statistiques relevant de deux états, A et B. Si les pourcentages obtenus par la famille politique $X$ étaient identiques dans chaque entité régionale du pays A et différents de ceux, eux-mêmes identiques, obtenus par la même famille politique dans chaque entité régionale du pays $\mathrm{B}, 100 \%$ de la variance totale relèverait de la variance inter-États. Si en revanche, les pourcentages obtenus par la famille politique $\mathbf{X}$ dans chaque entité régionale étaient parfaitement aléatoires par rapport à l'appartenance de cette entité à l'un des deux États, la variance inter-États serait nulle.

15 Le poids des déterminants nationaux dans les géographies électorales du scrutin européen apparaît le moins marqué pour la famille écologiste, traduisant l'existence de comportements politiques similaires parmi les classes moyennes post-modernes intellectuelles cosmopolites bien présentes à travers l'Europe dans les noyaux métropolitains et les « régions qui gagnent », surtout en Europe occidentale. La force de la base de gauche classique dans les anciens bassins industriels lourds, même si cette caractéristique tend à s'affaiblir sensiblement, ainsi que dans beaucoup de noyaux métropolitains explique aussi la variance inter-États un peu plus faible pour les partis sociaux-démocrates. La variance inter-États apparaît plus forte pour les partis de droite, plus « attrape-tout » et dont les géographies nationales sont en conséquence en général plus ubiquistes. Elle est maximale pour les partis de la droite extrême et pour les eurosceptiques, pour lesquels les facteurs politiques nationaux de leur succès ou de leur faiblesse apparaissent presque «naturellement » déterminants, puisqu'ils sont la base même de leur existence et de leur discours identitaire.

De manière générale, cette prééminence des déterminants nationaux doit évidemment sous-tendre toute interprétation des résultats. Le tableau 1 montre aussi que les évolutions des résultats d'une élection à l'autre sont encore plus sensibles à des considérations nationales que le niveau des résultats lui-même.

17 En outre, à la perception distanciée des enjeux politiques européens par les électeurs, s'ajoute un désenchantement par rapport à la construction européenne telle qu'elle est concrètement ressentie. Des taux de participation très nettement inférieurs à ceux enregistrés aux élections législatives, en particulier dans les nouveaux pays membres de l'Union européenne, en sont la preuve. Parmi les anciens membres de l'Union européenne, en dehors de ceux où le vote est ou était théoriquement obligatoire, le taux 
de participation aux élections européennes par rapport aux inscrits est de $42 \%$, contre $67 \%$ aux dernières législatives. Dans les nouveaux pays membres, il n'est que de $26 \%$ ( $51 \%$ aux législatives). Les "records" de faible participation sont enregistrés en Slovaquie, avec $12,7 \%$ des inscrits, et en République tchèque, avec $18,1 \%$. Même dans les pays où le vote est théoriquement obligatoire, le taux de participation n'est que de $59 \%$ aux européennes, contre $75 \%$ aux législatives. Seuls trois pays à vote théoriquement obligatoire maintiennent un taux de participation supérieur à $70 \%$ aux européennes : la Belgique ( $84 \%$ ), le Luxembourg (77 \%) et Malte (73\%).

Toutefois, le désenchantement par rapport à la situation économique et sociale générale et aux déficits politiques européens ne s'est pas traduit par une baisse du taux de participation entre 2009 et 2014 (tableau 2). Celui-ci tend globalement à se stabiliser, à un niveau il est vrai très bas, depuis le début des années 2000 (45,7 \% en 2004), après avoir fortement régressé de 63,0 \% en 1979 à 49,8 \% en 1999, parmi les pays membres de l'Union à l'époque.

Tableau 2 - Pourcentage de votes valables par rapport aux inscrits (dans certains pays, aux législatives, pourcentage de votes exprimés)

\begin{tabular}{|c|c|c|c|}
\hline & $\begin{array}{l}\text { Élections législatives précédant les } \\
\text { élections européennes de } 2014\end{array}$ & $\begin{array}{l}\text { Élections } \\
\text { européennes de } \\
2009\end{array}$ & $\begin{array}{l}\text { Élections } \\
\text { européennes de } \\
2014\end{array}$ \\
\hline France (a) & $53,3(2012)$ & 39,0 & 41,6 \\
\hline Belgique (b) & $84,3(2014)$ & 84,7 & 84,2 \\
\hline $\begin{array}{l}\text { Luxembourg } \\
\text { (b) }\end{array}$ & $91,2(2013)$ & 90,9 & 77,1 \\
\hline Pays-Bas & $74,6(2012)$ & 36,5 & 37,1 \\
\hline Allemagne & 70,7 (2013) & 42,3 & 47,3 \\
\hline Autriche & $74,4(2013)$ & 45,0 & 44,0 \\
\hline Danemark & 87,7 (2011) & 57,7 & 55,0 \\
\hline Suède & $84,6(2010)$ & 44,7 & 48,2 \\
\hline Finlande & $70,4(2011)$ & 38,5 & 41,0 \\
\hline Royaume-Uni & $65,1(2010)$ & 34,5 & 35,4 \\
\hline Irlande & $70,1(2011)$ & 56,1 & 51,0 \\
\hline Portugal & $58,0(2011)$ & 35,0 & 32,1 \\
\hline Espagne & 70,7 (2011) & 44,0 & 43,9 \\
\hline Italie (b) & 75,0 (2013) & 62,2 & 55,6 \\
\hline
\end{tabular}




\begin{tabular}{|c|c|c|c|}
\hline Malte (b) & $93,1(2013)$ & 81,0 & 73,1 \\
\hline Grèce (b) & 63,6 (2012) & 51,2 & 57,7 \\
\hline Chypre (b) & $80,9(2013, d)$ & 58,2 & 42,7 \\
\hline Pologne & $48,9(2011)$ & 24,1 & 23,1 \\
\hline Lituanie & $52,6(2012)$ & 20,4 & 44,7 \\
\hline Lettonie & $60,6(2011)$ & 52,3 & 29,9 \\
\hline Estonie & 63,6 (2011) & 43,6 & 36,4 \\
\hline $\begin{array}{l}\text { République } \\
\text { tchèque }\end{array}$ & $59,5(2013)$ & 28,1 & 18,1 \\
\hline Slovaquie & $58,1(2012)$ & 19,0 & 12,7 \\
\hline Hongrie & 61,2 (2014) & 36,0 & 28,8 \\
\hline Slovénie & 64,7 (2011) & 27,3 & 23,5 \\
\hline Croatie (c) & $54,3(2011)$ & 20,7 & 24,3 \\
\hline Roumanie & $41,7(2012)$ & 26,5 & 30,4 \\
\hline Bulgarie & $51,2(2013)$ & 38,4 & 33,7 \\
\hline UE 28 & 64,7 & 41,5 & 41,6 \\
\hline
\end{tabular}

(a) Sans les TOM ni les électeurs résidant à l'étranger.

(b) Vote théoriquement obligatoire, mais l'abstention n'est de fait plus sanctionnée.

(c) Élections européennes de 2013.

(d) Élections présidentielles. 
Illustration 2 - Taux de participation aux élections européennes de 2014 (en pourcentage des inscrits)

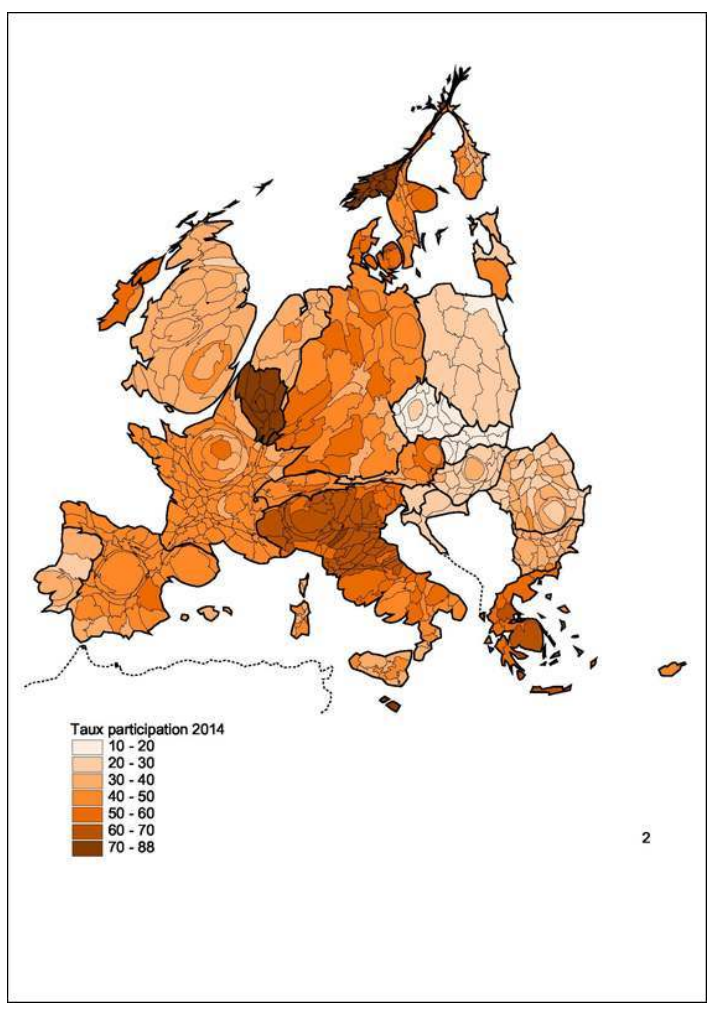

19 Au niveau régional, les taux de participation aux élections européennes les plus élevés sont souvent enregistrés dans les plus grandes métropoles (Paris, Athènes, Budapest, Prague, Stockholm, Helsinki, Lisbonne), ou du moins dans leurs périphéries aisées (Londres, ouest parisien, Vienne, Copenhague), que dans le reste des différents pays. C'est en Italie que les oppositions entre les taux de participation sont les plus frappantes au niveau régional, entre le nord et le centre d'une part, le sud de l'autre, Rome occupant une position intermédiaire.

\section{Des élections sanctions?}

Renforçant encore le poids des déterminants nationaux, il faut tenir compte du fait que les élections européennes interviennent dans chaque pays à des moments différents de la conjoncture politique. Leurs résultats expriment-ils dès lors une appréciation des politiques européennes ou plutôt des sanctions (ou approbations) des politiques des gouvernements en place?

On ne peut de ce point de vue dégager de tendance générale (tableau 3).

Dans presque tous les pays, la droite d'establishment non eurosceptique est majoritaire. Les exceptions sont la Grande-Bretagne et la Pologne, du fait du caractère très eurosceptique d'une part importante de la droite de gouvernement dans ces deux pays (respectivement les Conservateurs et le PiS, pour le moment dans l'opposition). On pourrait éventuellement y ajouter le FIDESZ hongrois, vu les ambiguïtés de son positionnement politique. La social-démocratie l'emporte en Italie et au Portugal. La gauche radicale en Grèce et en Irlande. 

recul d'une élection européenne à l'autre (de $44,8 \%$ à $34,3 \%$ ), bien qu'avec une série d'exceptions nationales. Quant aux sociaux-démocrates, ils progressent, mais peu (de $23,4 \%$ à $25,4 \%$ ).

Globalement, c'est clairement la droite extrême et identitaire qui profite le plus du désarroi face à la crise sociale et économique et d'un refus de l'Europe officielle ; mais elle a fait essentiellement campagne sur des thèmes nationaux. C'est elle qui progresse le plus en pourcentage et dans le plus grand nombre de pays. D'une élection européenne à l'autre, le FN progresse de 13,6 \% à 24,9 \% en France ; les Vrais Finlandais de 9,8 \% à 12,9\% en Finlande; les Démocrates suédois de $3,3 \%$ à $9,7 \%$; le FPÖ de $12,7 \%$ à $19,7 \%$ en Autriche; le Parti populaire danois de $15,3 \%$ à $26,6 \%$; l'UKIP de $16,7 \%$ à $27,5 \%$. En Pologne, Nowa Prawica obtient $7,2 \%$, sans parler du très conservateur et nationaliste PiS qui passe de $27,4 \%$ à $31,8 \%$. Mais la règle n'est toutefois pas absolue : JOBBIK stagne en Hongrie, où il est vrai l'électorat populiste de droite nationaliste peut se retrouver dans le FIDESZ; Ataka régresse fortement en Bulgarie, de même que la Parti de la Grande Roumanie, ou le Vlaams Belang en Flandre (mais ici aussi la NVA peut satisfaire l'électorat nationaliste flamand très à droite); le PVV s'affaiblit aux Pays-Bas. Hors Union, l'extrême-droite s'affaiblit en Suisse et en Norvège. L'extrême-droite ne perce ni en Italie, du moins si on n'y place pas le Mouvement 5 Étoiles, anti-système anarcho-populiste, ni en Allemagne, ni en Espagne. Au total cependant, à l'échelle de l'ensemble de l'UE, l'extrême-droite passe de 9,4 à 15,1\% d'une élection européenne à l'autre (de 15,6 \% à $22,3 \%$ si on y ajoute la droite classique eurosceptique).

A l'inverse, la gauche radicale ne profite que peu du mécontentement. Elle ne progresse que de $6,4 \%$ à $7,9 \%$ (de $14,6 \%$ à $15,9 \%$ si on y ajoute les écologistes). Néanmoins, la réaction de gauche est évidente dans les pays les plus touchés par la crise. Mais elle y profite à des partis alternatifs, plutôt issus de la société civile qu'inscrits dans les traditions de la gauche de filiation communiste. Ainsi, par rapport aux élections législatives de 2011, le Parti populaire au pouvoir en Espagne régresse de 44,6 \% à 26,7 \%, ce qui ne profite pas au Parti socialiste (qui régresse lui aussi de $28,7 \%$ à $23,5 \%$ ), un peu à Izquierda Unida (qui passe de $6,9 \%$ à $10,2 \%$, mais avec de nombreux partis régionalistes associés), mais surtout à Podemos, qui s'impose d'emblée avec $8,2 \%$. Il en va de même en Grèce : par comparaison avec les dernières élections législatives antérieures à la crise, en 2009, la droite de Nouvelle Démocratie régresse de $33,5 \%$ à $22,7 \%$ et le parti socialiste Pasok s'effondre de $43,9 \%$ à $8,0 \%$; Syriza, expression d'une nouvelle extrême-gauche, progresse en revanche de $4,6 \%$ à $26,6 \%$, alors que le mécontentement ne profite pas au Parti communiste grec KKE qui baisse de 7,5 \% à 6,1 \%. En Slovénie, Verjamem! (Je crois ! ), mouvement qui s'est affilié au groupe des Verts, recueille d'emblée 10,3\% des suffrages exprimés. Au Portugal en revanche, par rapport aux législatives de 2011, tant le Parti socialiste que le Parti communiste progressent (respectivement de $28,1 \%$ à $34,0 \%$ et de $7,9 \%$ à $13,7 \%$ ), à l'encontre du PPD/PSD qui baisse de $38,7 \%$ à $29,9 \%$ et en Italie, le mécontentement profite clairement au PD (qui passe de 29,5\% aux législatives de 2013 à $40,8 \%$ ), le mouvement populiste 5 Étoiles semblant entamer un déclin après son triomphe de 2013 (de $25,5 \%$ à $21,2 \%$ ).

En moyenne européenne, les effets de la crise se marquent donc principalement par des pertes de la droite de gouvernement et des gains de la droite extrême (respectivement $9,4 \%$ et $+5,7 \%$ ). À gauche, les résultats sont globalement plus stables: les sociauxdémocrates gagnent $1,9 \%$, tirés par leurs résultats italien, britannique, allemand, ainsi 
que roumain et portugais, mais avec des pertes spectaculaires pour certains partis sociaux-démocrates de gouvernement (en Grèce, - 20,9\%; en Croatie, - 20,0\%; en Slovénie, $-10,3 \%$; en République tchèque, - 8,9\%; en Slovaquie, - 8,3\%, ainsi qu'en France, - 17,2\%, avec les divers gauche, mais ici par rapport aux législatives de 2012), voire d'opposition (en Espagne, - 15,7 \%). Les Verts et l'extrême-gauche gagnent ensemble un modeste $1,3 \%$,

Tableau 3 - Résultats des élections européennes de 2014, par grande tendance politique (en \% des votes valables

\begin{tabular}{|c|c|c|c|c|c|}
\hline & \begin{tabular}{|} 
Gauche \\
radicale
\end{tabular} & Ecologistes & $\begin{array}{c}\text { Sociaux- } \\
\text { démocrates }\end{array}$ & \begin{tabular}{|c} 
Libéraux, \\
démocrates- \\
chrétiens \\
conservateurs
\end{tabular} & $\begin{array}{c}\text { Droite } \\
\text { eurosceptique ot } \\
\text { etroite radicale } \\
\text { detou europhobe }\end{array}$ \\
\hline France (a) & 8.0 & 89 & 17,1 & 36,6 & 25.0 \\
\hline Belgique & 3.8 & 11,1 & 19,1 & 40,8 & 24,9 \\
\hline Luxembourg & 7.3 & 19,2 & 0.8 & 52,5 & 9.4 \\
\hline Pays-Bas & 13,9 & 8,7 & 9,4 & 42,8 & 21,5 \\
\hline Allemagne & 7,6 & 14,0 & 27.3 & 40.4 & 29 \\
\hline Autriche & 0,0 & 14,5 & 24,1 & 35,1 & 24.1 \\
\hline Danemark & 0,0 & 11,0 & 19,1 & 35,2 & 34.7 \\
\hline Suede & 6,3 & 17,5 & 29,7 & 36,1 & 10,0 \\
\hline Finlande & 9,7 & 10,0 & 12.3 & 54,2 & 13,2 \\
\hline Royaume-Uni & 1,0 & 10,7 & 24.9 & 6.6 & 52,8 \\
\hline Irlande & 29.4 & 5.2 & 11.9 & 28,9 & 24,6 \\
\hline Portugal & 21,0 & 11.9 & 34.8 & 30.1 & 1,8 \\
\hline Espagne & 20.8 & 7.8 & 23,9 & 43,8 & 0,3 \\
\hline Italie & 4,0 & 0,9 & 40,8 & 23,3 & 81.0 \\
\hline Malte & 0,0 & 2,9 & 53,4 & 40,1 & 3,1 \\
\hline Grèce & $3+3$ & 1,7 & 159 & 28,1 & 15.9 \\
\hline Chypre & 27.0 & 09 & 18,6 & 37,7 & 2,7 \\
\hline Pologne & 0,0 & 0,5 & 9,4 & 42.5 & 47,5 \\
\hline Lituanie & 0,0 & 3.6 & 17,3 & 88.2 & 30.2 \\
\hline Lettonie & 1,5 & 6,4 & 17.2 & 51.5 & 22,1 \\
\hline Estonie & 0,0 & 13.2 & 13,6 & 62.4 & 5,3 \\
\hline $\begin{array}{l}\text { République } \\
\text { tchèque }\end{array}$ & 11,5 & 25 & 14.2 & 92.8 & 17.4 \\
\hline Slovaquie & 1,8 & 0,9 & 24,3 & 52,1 & 14,7 \\
\hline Hongrie & 0,0 & 12.2 & 20,7 & 51.5 & 14.7 \\
\hline Slovénie & 5.3 & 13.1. & 8.1 & 63.2 & 4.4 \\
\hline Croatie & 3.9 & 11.6 & 22.0 & 52,3 & 7,3 \\
\hline Roumanie & 0,2 & 1,5 & 87.1 & 50,0 & 4.8 \\
\hline Bulgarie & 0,6 & 0,9 & 23,1 & 55,8 & 17,0 \\
\hline UE 28 & 79 & 8,0 & 25,4 & 34,3 & 2.3 \\
\hline dont UE 15 & 9.0 & 8,7 & 26,1 & 32.6 & 22.1 \\
\hline $\begin{array}{c}\text { dont nouveaux } \\
\text { membres }\end{array}$ & 1,4 & 3.7 & 21,1 & 48,2 & 22,9 \\
\hline Suisse & 0,8 & 13,9 & 18,5 & 14,0 & 50,1 \\
\hline Norvège & 5,2 & 2.8 & 30,8 & 37.5 & 21.9 \\
\hline
\end{tabular}

(a) Sans les TOM ni les électeurs résidant à l'étranger. Le chiffre en gras souligne la tendance majoritaire. Les couleurs indiquent la tendance par rapport à 2009 (progression de plus de $5 \%$ en rouge ; de 1 à $5 \%$ en orange ; diminution de 1 à $5 \%$ en bleu clair ; de moins de $5 \%$ en bleu foncé)

Examinons maintenant, famille par famille, les géographies électorales qui se dégagent des élections européennes de 2014 (complétées par les législatives de 2013 et de 2011 pour la Norvège et la Suisse). Une série de constats en seront tirés, mais il est bien évident que l'objectif de cet article est avant tout de rendre compte des grandes leçons de géographie électorale qui se dégagent des dernières élections européennes, et non de procéder à une analyse approfondie des déterminants des comportements électoraux régionaux à l'intérieur de chaque pays européen. Pour de telles analyses, on peut se référer à une importante littérature (voir e.a. pour les déterminants des comportements électoraux Derivry et Dogan, 1985 ; Smith, 1988 ; Dogan, 1996 ; Agnew, 1996, 2002 ; Johnston \& Pattie, 2000 et, avec une certaine prudence Todd, 1991 ; et, pour l'analyse plus spécifiquement géographique, Van Laer, 1984 ; Bussi, 1998 ; Vandermotten, Colard, Delwit, 1996 ; David \& Van Hamme, 2011; Van Hamme, 2008, 2012; avec une synthèse dans Vandermotten et Vandeburie, 2011). 


\section{La carte de l'extrême-gauche et des écologistes}

La carte de ces partis, regroupés parce qu'ils proposent une rupture de gauche par rapport à la social-démocratie, n'est pas de lecture aisée (illustration 3), dans la mesure où elle additionne des géographies différentes, celles des écologistes (illustration 4) et de l'extrême-gauche (illustration 5), elle-même constituée d'une extrême-gauche classique, issue de la tradition communiste, de nouveaux mouvements d'extrême-gauche, nés au sein de la société civile, et de partis régionalistes de gauche, rattachés à la Gauche unitaire européenne, bien que sans liens avec l'histoire internationaliste du mouvement ouvrier, tel le Sinn Fein (d'autres le sont au groupe Verts/ALE, en particulier les régionalistes écossais et gallois).

Illustration 3 - Pourcentage des votes valables obtenu par les partis écologistes et d'extrêmegauche (2014)

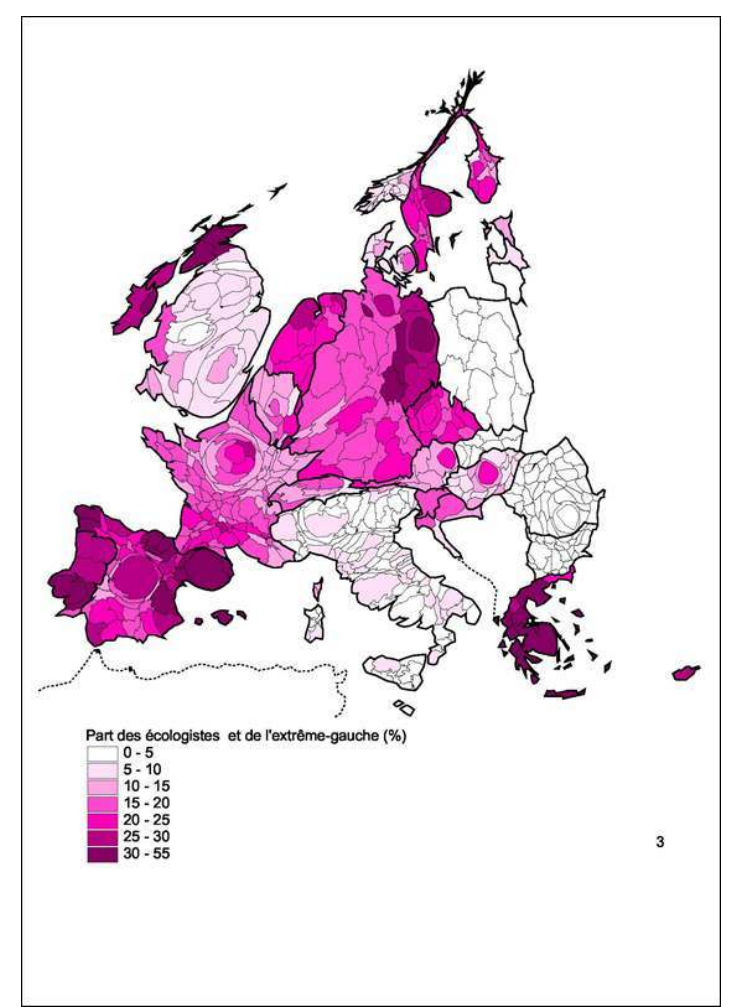


Illustration 4 - Pourcentage des votes valables obtenu par les partis rattachés à la famille écologiste (2014)

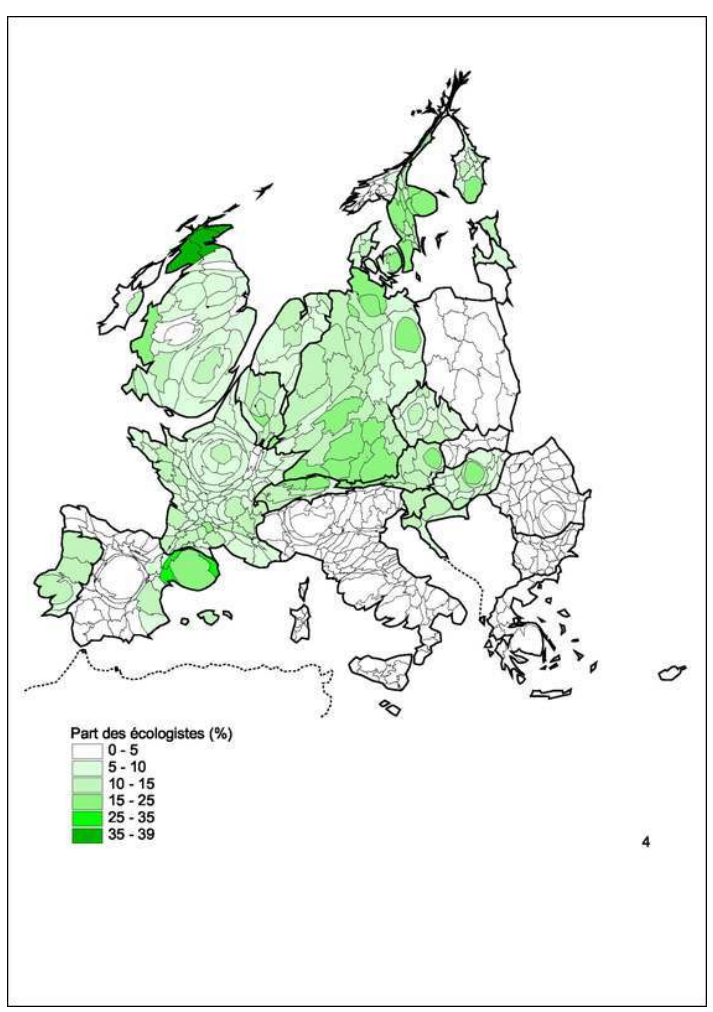

Illustration 5 - Pourcentage des votes valables obtenu par les partis rattachés à la famille d'extrême-gauche (2014)

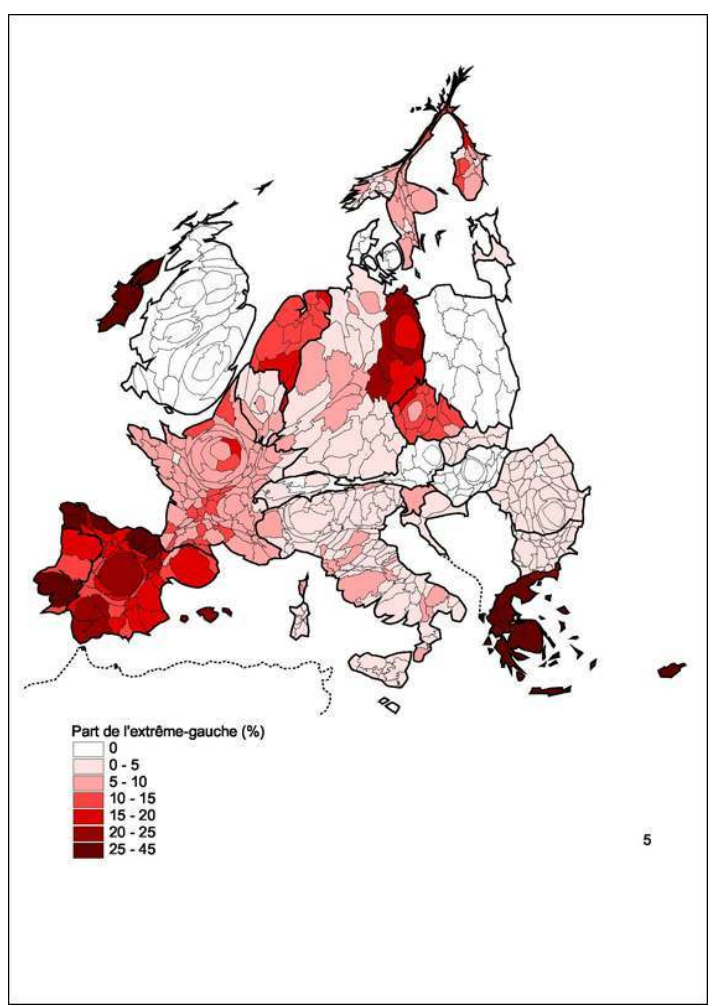



l'Europe périphérique, méditerranéenne et centre-orientale. Quand ils apparaissent en Europe centre-orientale, c'est plutôt sous la forme de mouvements alternatifs que sous celle de partis verts classiques (Verjamem!, en Slovénie; Développement durable, en Croatie ; La politique peut être différente, en Hongrie). L'écologie politique classique est donc avant tout affaire des pays du centre européen, Allemagne en tête, et de la Scandinavie. Dans tous les cas, l'écologie politique a aussi une géographie centrale aux échelles nationales, elle est sur-représentée dans les grandes villes dotées d'un important secteur non marchand et culturel (Stockholm, Copenhague, Hambourg, Brême, Berlin, Amsterdam, Londres, Dublin, Paris, Vienne, Zurich), y compris en Europe centre-orientale (Prague, Budapest), ou dans des zones prospères peuplées de populations aisées, culturellement ouvertes, mais étrangères aux formes traditionnelles d'encadrement $\mathrm{du}$ mouvement socialiste (le Brabant wallon périurbain riche au sud de Bruxelles; le sud catholique et prospère de l'Allemagne, fief historique de la CDU-CSU ; le Grand-Duché de Luxembourg). Barcelone et la Catalogne combinent écologisme politique de région urbaine prospère et alliance entre l'écologisme et des mouvements régionalistes de gauche.

À l'inverse, la carte de l'extrême-gauche "classique » est celle de zones périphériques. Elle n'a en tout cas pratiquement plus de points communs avec celle des bastions ouvriers historiques des partis communistes, telle qu'on pouvait l'observer dans les décennies postérieures à la Seconde Guerre mondiale, d'autant qu'on l'a vu, elle est dans plusieurs pays dominée par des mouvements sans filiation avec la famille communiste historique : la Grèce avec Syriza ; l'Espagne, avec Podemos, qui dépasse IU à Madrid ; l'Irlande avec le Sinn Fein; le parti socialiste (de gauche) néerlandais. Certes, quelques bastions traditionnels, tout relatifs, de l'extrême-gauche «historique " subsistent (l'Andalousie, Barcelone, le Pays basque, les Asturies en Espagne, mais il faut tenir compte dans ces trois derniers cas, ainsi qu'en Galice, des alliances avec des partis régionalistes de gauche ; ou encore, très affaiblis, la banlieue est de Paris, le Pas-de-Calais, les anciens bassins wallons, où le Parti du Travail de Belgique - PTB - prend de l'importance, mais lui non plus n'est pas lié à l'ancien parti communiste). L'image d'une extrême-gauche de périphérie est claire en Scandinavie (le nord vs. le sud), ainsi qu'en Allemagne, et là elle l'est doublement (l'est vs. l'ouest; le reste de l'Allemagne de l'est vs. ses «centres", Berlin et la Saxe), même si Die Linke détient aussi quelques positions dans d'anciens bastions ouvriers sociaux-démocrates urbains et industriels de l'ouest (Hambourg, Brême, la Ruhr, la Sarre). En Europe centre-orientale, dans le seul pays outre l'Allemagne de l'est où l'extrême-gauche de filiation communiste est bien représentée, la République tchèque, sa géographie est là aussi périphérique.

\section{Les partis du consensus européen}

31 Parmi les partis qui s'inscrivent dans la logique dominante de la construction européenne et sont associés dans la mise en œuvre des compromis qui en fondent la gouvernance, nous distinguerons successivement ceux qui relèvent de la famille social-démocrate et ceux de centre-droit. 


\section{La social-démocratie}

Illustration 6- Pourcentage des votes valables obtenu par les partis rattachés à la famille socialedémocrate (2014)

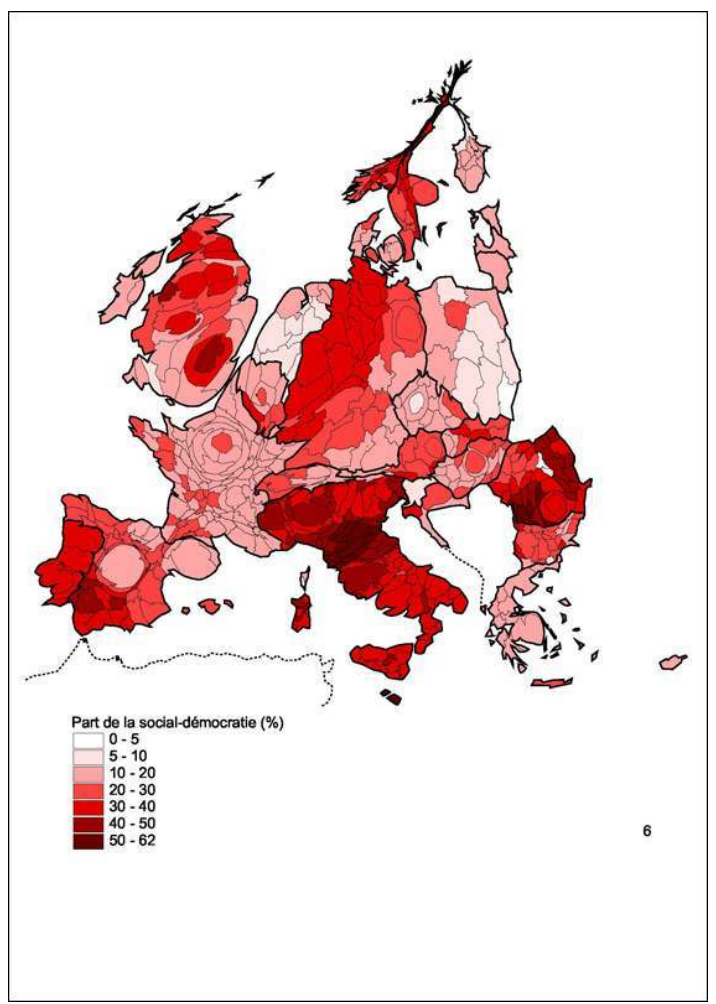

32 Au-delà des déterminants nationaux (la force d'encadrement héritée de l'ancien PCI évidente dans les anciens bastions rouges de la Troisième Italie - et l'effondrement du berlusconisme en Italie; les capacités organisationnelles et les liens avec le mouvement syndical assurant le maintien du poids socialiste en Belgique francophone, en Allemagne, en Suède; les héritages clientélistes dans la vieille Roumanie non métropolitaine ; etc.), les forces et les faiblesses régionales de la social-démocratie relèvent de différentes logiques, soit héritées de temps longs et tant bien que mal maintenues à travers les structures d'encadrement, soit plus récentes. Leurs effets se superposent parfois. Ceci ne permet pas de proposer une interprétation globale et univoque de la carte. C'est ce qui rend aussi très délicat pour les partis sociaux-démocrates l'affirmation d'une ligne politique claire: ils doivent satisfaire différents types d'électorats, dont les positionnements sociétaux et idéologiques peuvent être opposés, par exemple envers l'immigration ou la post-modernité socio-culturelle.

On peut citer, parmi les facteurs d'une implantation privilégiée :

- les héritages des encadrements ouvriers historiques dans les bassins de vieille industrialisation, en particulier ceux d'industrie lourde et minière et spécifiquement, en pays catholique, ceux où la structuration politique du monde ouvrier a débuté avant la publication de l'encyclique De Rerum Novarum (bassins industriels wallons, Ruhr, anciens bassins industriels anglais, etc.); 
- les héritages d'une gauche rurale anticléricale, liée aux structures agraires du passé, comme la grande propriété générant luttes sociales et réactions anticléricales (Andalousie) ou les pays de métayage (nord-ouest du Massif central) ;

- en creux, les héritages des encadrements par l'église catholique et les forces sociales traditionalistes (Flandre vs. Wallonie; nord protestant vs. sud catholique en Allemagne; Pologne "nouvelle» de l'ouest, repeuplée et avec une agriculture socialisée après la Seconde Guerre mondiale sur des «terres vierges ", vs. Pologne " autochtone », ancrée dans le terroir et le conservatisme religieux, où le régime socialiste n'a pas pu abolir la petite propriété foncière) ;

- l'émergence d'une nouvelle clientèle social-démocrate intellectuelle, liée au monde du non marchand et aux services publics, en général bien présente dans les cœurs des grandes métropoles (Paris, Londres, Bruxelles, Amsterdam, Milan, Rome, Vienne, Budapest, etc.). Toutefois, ces grandes métropoles peuvent apparaître en creux pour le vote socialdémocrate quand cette nouvelle classe moyenne intellectuelle en voie de prolétarisation a basculé vers de nouveaux mouvements d'extrême-gauche ou écologistes : c'est le cas en Espagne (Madrid, Barcelone), outre, de manière générale, en Grèce.

\section{Le centre-droit et la droite d'establishment}

Illustration 7- Pourcentage des votes valables obtenu par les partis rattachés aux familles libérale, chrétienne-démocrate et conservatrice non eurosceptique (2014)

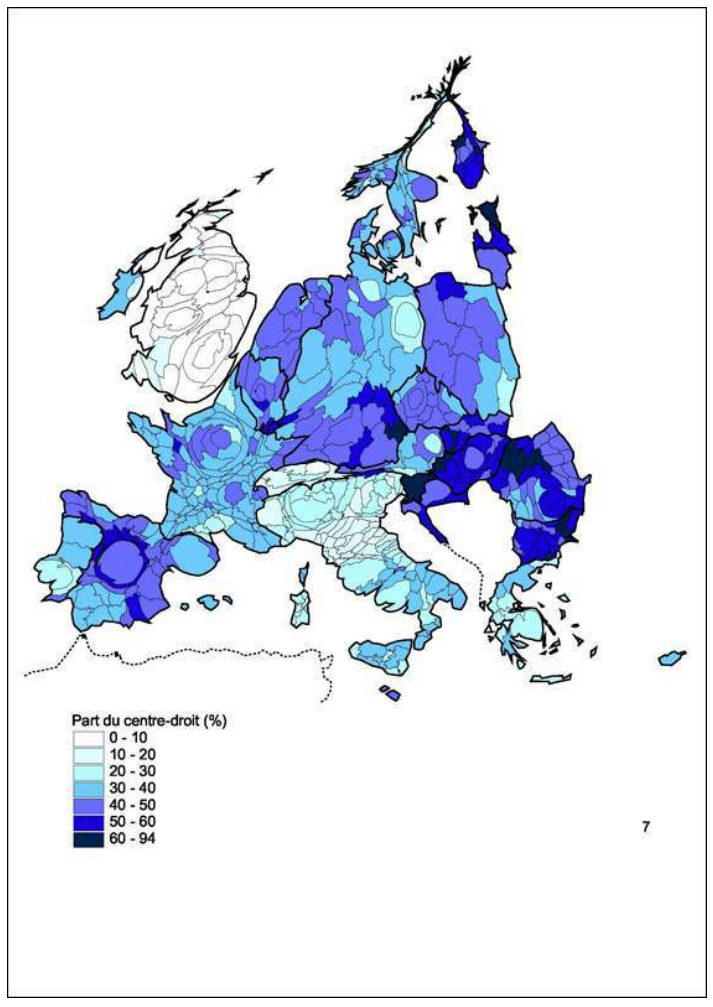

La carte du centre-droit d'establishment s'interprète finalement plus aisément «en creux ", à partir de ses zones de faiblesse, et ceci à différentes échelles.

Aux échelles nationales, elle est faible là où sa base électorale sociologique est récupérée par un ou des partis de droite eurosceptique (Grande-Bretagne, Suisse), ou là où elle s'est effondrée suite à un séisme politique (l'Italie, avec le laminage du berlusconisme; la 
Grèce, suite à la crise). Dans ce second cas, la droite classique conserve des positions meilleures dans les zones périphériques, où les comportements politiques restent plus stables et mieux encadrés par le clientélisme local (Mezzogiorno, bien que cela soit là aussi que la concurrence anti-système du Mouvement 5 Étoiles soit la plus forte; nord de la Grèce, Péloponnèse).

Aux échelles locales, le centre-droit apparaît plutôt en creux dans les grandes métropoles, plus favorables au centre-gauche d'establishment ou à la gauche radicale ou écologiste : Lisbonne, Madrid, Barcelone, Dublin, Berlin, Vienne, Budapest, Zagreb, Athènes. Mais la règle n'est pas absolue : certaines métropoles, qui combinent force des élites du secteur marchand internationalisé et force de celles relevant du secteur non marchand administratif et intellectuel, plus une importante population d'origine immigrée favorable à la gauche, peuvent donner simultanément de bons résultats à la droite classique et à la gauche, en fonction des quartiers ou des types de banlieue : c'est le cas à Paris, où tant la gauche que la droite classique font de relativement bons résultats, la concurrence du Front national étant moindre qu'ailleurs en France et la droite étant en outre particulièrement forte dans les banlieues aisées de l'ouest francilien; à Lyon; à Bruxelles, où les partis de centre-droit - mais aussi les écologistes - sont forts dans les quartiers aisés de l'est et du sud-est et en banlieue, alors que les partis de gauche classique dominent dans les quartiers centraux et d'immigrés ; à Stockholm ; à Helsinki ; à Varsovie, où la droite moderne l'emporte sur la droite eurosceptique traditionaliste; à Prague, Bucarest, Sofia. Dans ces dernières villes, le poids des nouveaux milieux d'affaire l'emporte sur les forces de l'ex-nomenklatura restées liées à l'appareil administratif et souvent reconverties à la social-démocratie.

Toujours en creux, nous retrouvons aussi les oppositions « historiques » en Allemagne, entre régions catholiques (au sud) et protestantes (ou d'ancienne industrialisation lourde) (au nord); en péninsule ibérique, entre le sud historiquement marqué par la grande propriété agricole et les zones de petite propriété ; la force plus grande du centre-droit dans les zones «modernes » en Pologne (l'ouest) ou en Roumanie (la Transylvanie), face à des zones plus traditionalistes (l'est ultra-conservateur de la Pologne) ou restées mieux encadrées par les héritiers de la nomenklatura (la Moldavie et la Valachie roumaines).

\section{L'extrême-droite et les eurosceptiques}


Illustration 8 - Pourcentage des votes valables obtenu par les partis rattachés aux familles conservatrice eurosceptique et à la droite extrême, populiste et/ou identitaire (2014)

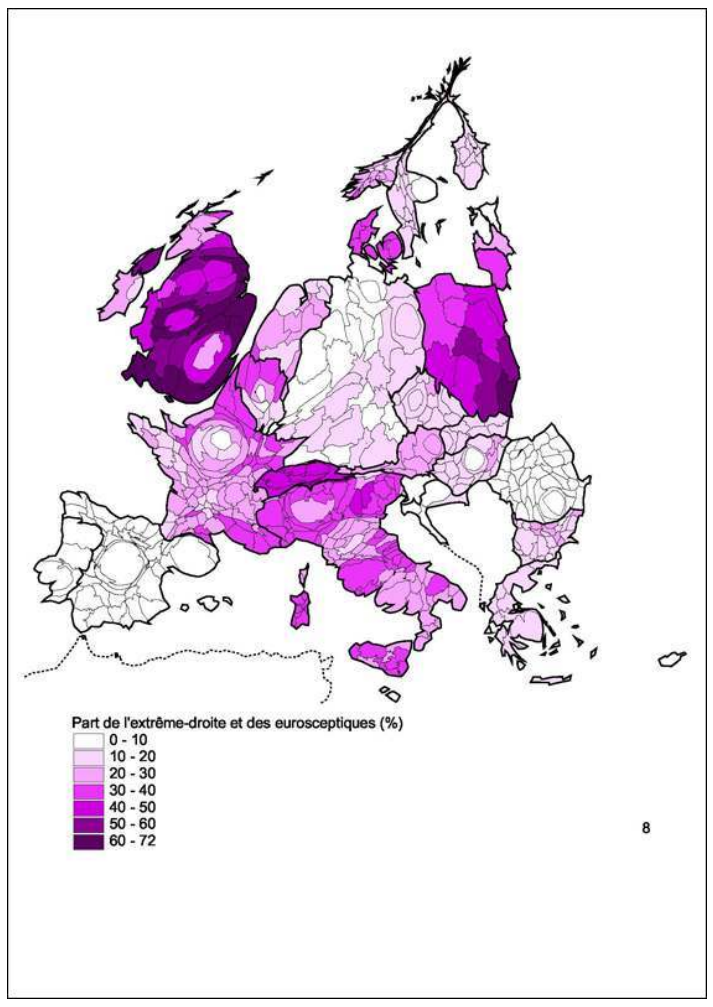

Illustration 9- Pourcentage des votes valables obtenu par les partis rattachés à la famille conservatrice eurosceptique (2014)

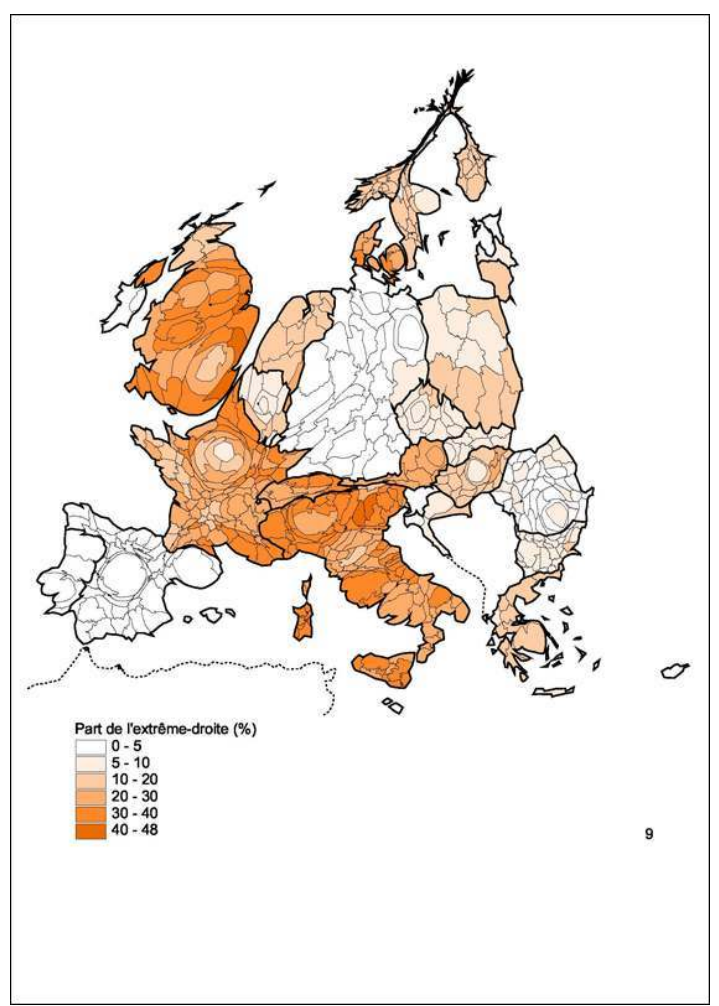


Illustration 10 - Pourcentage des votes valables obtenu par les partis rattachés à la droite extrême, populiste et/ou identitaire (2014)

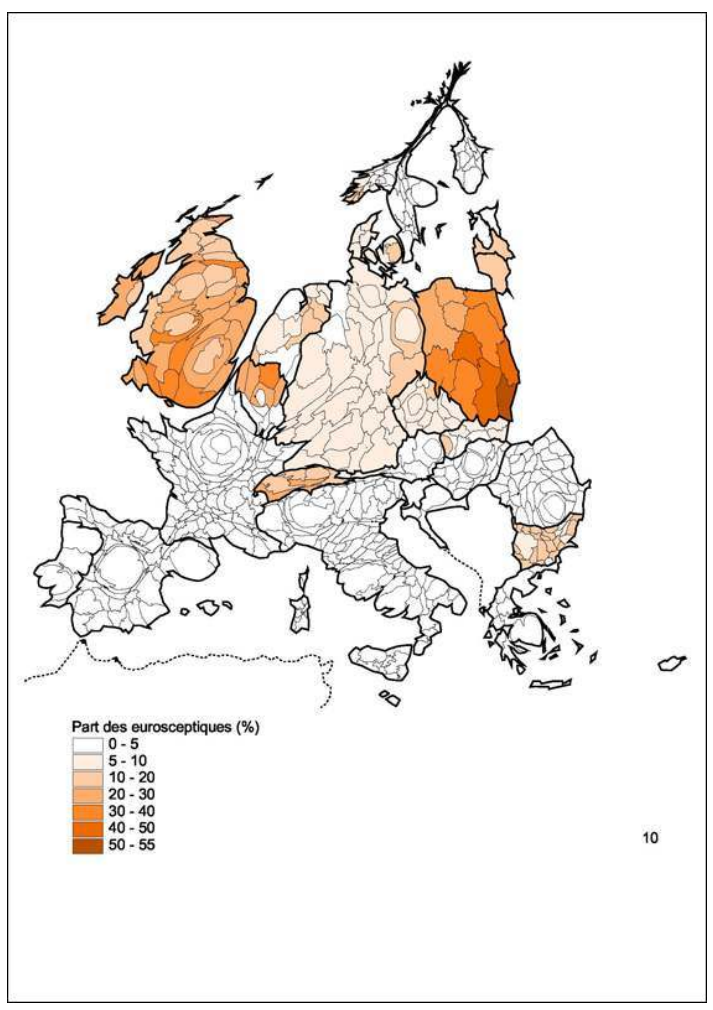

Tout en ne prétendant évidemment en aucune manière assimiler un parti tel que les Conservateurs britanniques à une quelconque forme d'extrême-droite, nous avons rassemblé ici l'ensemble des partis qui refusent les formes prises par la construction européenne sur la base d'un rejet de l'intégration sociale et d'un approfondissement des règles communes, d'une défense radicale des préférences nationales (éventuellement doublée d'atlantisme), voire qui souhaitent sortir de ce processus de construction (illustration 8). Nous avons néanmoins aussi cartographié séparément les partis eurosceptiques liés à l'establishment (pour l'essentiel ceux qui forment le groupe ECR au Parlement européen) (illustration 9) et les autres, qui apparaissent au contraire en rupture avec les positions de leurs establishments nationaux (illustration 10).

Certains pays semblent échapper à la poussée de ces tendances : l'Allemagne, sans doute en fonction du leadership qu'elle exerce sur la construction européenne - mais pour combien de temps encore quand on voit la montée en puissance de l'AfD - , mais aussi en réponse à la vigueur qui y a pris la dénazification politique après la Seconde Guerre mondiale, ce qui la distingue clairement de ce point de vue de l'Autriche; la péninsule ibérique, où la référence au franquisme et au salazarisme empêche le développement de l'extrême-droite en tant que telle ; la Roumanie, où le parti de la Grande Roumanie est en très net repli. Ailleurs, elles prennent des formes très diversifiées, qui ne permettent pas de parler d'un mouvement d'ensemble à l'échelle européenne : euroscepticisme marqué des Conservateurs et europhobie très conservatrice, couplée avec des attitudes xénophobes et anti-immigration de l'UKIP en Grande-Bretagne; traditionalisme nationaliste du PiS et des mouvements d'extrême-droite chrétienne en Pologne ; Front national en France, associant populisme contemporain sur fond d'islamophobie et traditions de la droite ultra-conservatrice; phénomène des Ligues, en perte de vitesse, 
mais développement de l'anarchisme de droite du Mouvement 5 Étoiles en Italie ; droite extrême conservatrice de l'UDC en Suisse et du FPÖ en Autriche ; Parti populaire danois ; Vrais Finnois; etc. Notons que la carte est affaiblie en Hongrie par le fait que le FIDESZ garde une place, ambigüe, au sein du PPE, malgré son autoritarisme et sa xénophobie anti-Roms. En Flandre, l'extrême-droite fascisante a été fortement affaiblie par le transfert massif des électeurs du Vlaams Belang vers la très à droite NVA, liée à une partie de l'establishment économique flamand et qui a rallié le groupe eurosceptique dominé par les Conservateurs britanniques.

De manière générale, l'europhobie et l'extrême-droite ont une géographie qui est plutôt celle de régions de perdants (avec les exceptions notables de la Flandre et de l'Italie du nord, régions gagnantes mais avec un électorat qui refuse la solidarité nationale sur une base culturelle identitaire). Il s'agit par exemple de vieilles régions ouvrières en rupture d'encadrement socio-politique, comme le nord et l'est de la France, ou des anciens bassins anglais. On enregistre aussi de bons résultats pour la droite extrême dans des régions de populations fragilisées, âgées, parfois aisées mais inquiètes ou aigries (périurbain profond, en frange externe de la Région parisienne ; sud méditerranéen français ; sud de l'Angleterre). À l'inverse, ces forces sont moins présentes dans les grandes métropoles, sociologiquement plus ouvertes et plus habituées à la confrontation des cultures malgré les concentrations de populations d'origine immigrée qui y prévalent, ou peut-être du fait de celles-ci, et où s'imposent soit les partis d'establishment, soit la gauche classique et plus encore, du moins en termes relatifs, alternative. Toutefois, la géographie est différente quand il s'agit d'une droite eurosceptique d'establishment comme les Conservateurs britanniques (ou, moins eurosceptique, la NVA en Flandre) plutôt que d'une droite extrême populiste: sa répartition est alors plus ubiquiste, avec les meilleurs résultats dans les zones bourgeoises aisées.

\section{Conclusions}

Tableau 4. Résultats électoraux aux Européennes de 2009 en pourcentage des électeurs inscrits

\begin{tabular}{|l|l|l|l|}
\hline & $\begin{array}{l}\text { Gauche } \\
\text { radicale et } \\
\text { écologistes }\end{array}$ & $\begin{array}{l}\text { Partis d'establishment pro- } \\
\text { européens (sociaux-démocrates, } \\
\text { libéraux, démocrates-chrétiens et } \\
\text { conservateurs) }\end{array}$ & $\begin{array}{l}\text { Droite eurosceptique et } \\
\text { droite radicale et/ou } \\
\text { europhobe }\end{array}$ \\
\hline France (a) & 7,0 & 22,4 & 10,4 \\
\hline Belgique (b) & 12,6 & 50,5 & 21,0 \\
\hline $\begin{array}{l}\text { Luxembourg } \\
\text { (b) }\end{array}$ & 20,4 & 49,5 & 7,2 \\
\hline Pays-Bas & 8,4 & 19,4 & 8,0 \\
\hline Allemagne & 10,2 & 32,0 & 4,7 \\
\hline Autriche & 6,4 & 26,1 & 10,6 \\
\hline
\end{tabular}




\begin{tabular}{|c|c|c|c|}
\hline Danemark & 6,0 & 29,9 & 19,1 \\
\hline Suède & 11,4 & 31,7 & 4,8 \\
\hline Finlande & 8,1 & 27,3 & 5,4 \\
\hline Royaume-Uni & 4,1 & 11,2 & 18,7 \\
\hline Irlande & 17,6 & 20,8 & 12,5 \\
\hline Portugal & 10,6 & 20,8 & 0,6 \\
\hline Espagne & 12,5 & 29,7 & 0,1 \\
\hline Italie (b) & 2,7 & 35,6 & 17,2 \\
\hline Malte (b) & 2,2 & 68,4 & 2,3 \\
\hline Grèce (b) & 20,7 & 25,4 & 9,2 \\
\hline Chypre (b) & 11,9 & 24,0 & 1,1 \\
\hline Pologne & 0,1 & 12,0 & 11,0 \\
\hline Lituanie & 1,6 & 29,3 & 13,8 \\
\hline Lettonie & 2,4 & 20,6 & 6,8 \\
\hline Estonie & 4,8 & 27,6 & 1,9 \\
\hline $\begin{array}{l}\text { République } \\
\text { tchèque }\end{array}$ & 3,8 & 10,3 & 3,1 \\
\hline Slovaquie & 0,4 & 9,7 & 1,9 \\
\hline Hongrie & 3,5 & 20,8 & 4,2 \\
\hline Slovénie & 4,5 & 16,7 & 1,0 \\
\hline Croatie & 3,8 & 18,1 & 1,8 \\
\hline Roumanie & 0,5 & 26,7 & 1,5 \\
\hline Bulgarie & 0,5 & 26,6 & 5,7 \\
\hline UE 28 & 6,6 & 24,8 & 9,3 \\
\hline dont UE 15 & 8,2 & 26,8 & 10,2 \\
\hline $\begin{array}{l}\text { dont nouveaux } \\
\text { membres }\end{array}$ & 1,3 & 18,2 & 6,0 \\
\hline
\end{tabular}

(a) Sans les TOM ni les électeurs résidant à l'étranger. 
(b) Vote obligatoire, mais l'abstention n'est de fait plus sanctionnée.

41 Si certaines conclusions générales peuvent être tirées de l'analyse régionale des résultats, par exemple quant aux conséquences de la désespérance sociale dans les régions en difficulté, jointe aux faillites des systèmes politiques traditionnels d'encadrement dans des régions où ceux-ci étaient historiquement puissants, ou quant à celles du désarroi des classes populaires, mais aussi de personnes aisées, souvent âgées, par rapport au cosmopolitisme entraîné par la mondialisation, elles doivent toutefois rester cadrées par la lecture des situations nationales et la connaissance des panoramas politiques et partisans propres à chaque pays.

42 La géographie des résultats des élections européennes reste avant tout celle d'une addition d'élections nationales, du moins dans les niveaux et les évolutions des forces partisanes. Ce constat rejoint la thèse de R. Harmsen (2005), quant à la relative stabilité des structures partisanes nationales, les partis nationaux d'establishment ayant domestiqué les enjeux européens, de sorte que la construction européenne ne serait pas accompagnée d'une formation d'un système partisan européen.

43 En ce qui concerne les logiques sous-tendant la répartition régionale des scores électoraux en revanche, quelques grandes constantes peuvent être dégagées: effets politiques, culturels et sociaux des situations métropolitaines, favorisant les votes d'ouverture socio-culturelle aux dépens des votes de repli identitaire; impacts de la vieille industrialisation, générant des inerties en faveur de la gauche classique, mais qui peuvent basculer vers une droite extrême de repli en cas d'effondrement des structures d'encadrement traditionnelles; rôle des forces historiques de l'encadrement par l'Église, en pays catholique et en particulier dans les zones rurales ou qui le sont longtemps restées ; oppositions centre - périphérie ; etc.

Ces élections européennes paraissent en outre être considérées comme moins importantes que les élections législatives par de larges fractions de l'électorat, ce dont témoignent les taux élevés d'abstention. Si l'on rapporte les votes émis au nombre d'inscrits et non au chiffre des votants, force est de constater que les partis défendant les formes dominantes de l'intégration européenne n'obtiennent que de l'ordre d'un quart d'adhésion (sauf dans les pays où le vote est théoriquement obligatoire), tout au plus de l'ordre du tiers parmi les "bons élèves » civiques de la classe européenne (Italie, Allemagne, Suède) (tableau 4). Les forces critiques de gauche mobilisent moins de $7 \%$ de l'électorat potentiel (essentiellement dans les anciens États membres), celles de droite un peu moins de $10 \%$. On est clairement loin d'une forte adhésion et mobilisation citoyennes. Ce désenchantement est particulièrement vif parmi les électorats des nouveaux pays membres, ainsi qu'en Grande-Bretagne, seul pays européen où l'opposition eurosceptique et europhobe de droite l'emporte nettement sur les votes en faveur des forces pro-européennes d'establishment (18,7\% des inscrits, contre $11,2 \%$; l'autre pays le plus eurosceptique étant la Pologne, $11,0 \%$ contre 12,0 \%). 


\section{BIBLIOGRAPHY}

Agnew J.A., 1996. Mapping politics: How context counts in electoral geography. Political geography, 15, 2, p. 129-146.

Agnew J.A., 2002. Place and politics in Modern Italy. Chicago, University of Chicago Press.

Brechon P., 2002. Les grandes enquêtes internationales (eurobaromètres, valeurs, ISSP) : apports et limites. L'année sociologique, 52, 1, p. 106-130.

Brustler G., Deloy C., Escalona F., 2014. Les familles politiques aux élections européennes de mai 2014 : un bilan. Fondation Robert Schuman Policy, Paper 319.

Bussi M., 1998. Éléments de géographie électorale à travers l'exemple de la France de l'ouest. Publication de l'Université de Rouen.

Coosemans T., 2014. Les résultats des élections européennes de mai 2014 dans les États membres. Courrier hebdomadaire du CRISP, 2221-222, Bruxelles,

David Q., Van Hamme G., 2011. Pillars and electoral behavior in Belgium: the neighborhood effect revisited. Political geography, 30, 5, p. 250-262.

Derivry D., Dogan M., 1985. Religion, classe et politique. Revue française de Science politique, 36, 2, p. $157-181$

Dogan M., 1996. Classe, religion, parti : triple déclin dans les clivages électoraux en Europe. Revue internationale de politique comparée, p. 515-540.

Harmsen R., 2005. L'Europe et les partis politiques nationaux : les leçons d'un non-clivage. Revue internationale de politique comparée, 12, 1, p. 77-94.

Ingelhart R., 1977. The Silent Revolution. Changing Values and Political Styles Among Western Publics. Princeton, Princeton University Press.

Ingelhart R., 2007. Postmaterialist Values and the Shift from Survival to Self-Expression Values. In Russel J., Dalton R.J. \& Klingeman H.D., Oxford Handbook of Political Behavior, p. 222-239.

Johnston R., Pattie C.J., 2000), "People who talk together vote together": an exploration of contextual effects in Great Britain. Annals of the Association of American Geographers, 90, 1, p. 41-66.

Lipset S.M., Rokkan S. (eds.) (1967, [trad. franç., 2008]). Party Systems, and Voter Alignments: Cross National Perspectives. New York, The Free Press [Structures de clivages, systèmes de partis et alignement des électeurs : une introduction, Bruxelles, Editions de l'Université de Bruxelles].

Rokkan S., 1970. Citizens, Elections, parties. Approaches to the Comparative Study of the processes of development. Oslo, Universitetsforlaget.

Seiler D.L., 2003. Les partis politiques en Occident - sociologie du phénomène partisan. Paris, Ellipses.

Smith J.L., 1988. Clivage sociaux, partis politiques et constants géographiques. Géographie sociale, 7, p. 3-43.

Tilburg University \& Gesis Leibniz Institute For The Social Sciences, 1999 \& 2008. EVS variable reports.

Todd E., 1991. L'invention de l'Europe. Paris, Le Seuil. 
Trechsel A.H., Mair P., 2009. When parties (also) position themselves : an introduction to the EU Profiler. EUI Working paper RSCAS 65, Florence, European Institute, Robert Schuman Centre for Advanced Studies, European Union Democracy Observatory, www.eui.eu/RSCAS/Publications

Vandermotten C., 2011 (2 $2^{\mathrm{ème}}$ éd.,). Territorialités et politique. Bruxelles, Éditions de l'Université de Bruxelles.

Vandermotten C., Colard A., Delwit P., 1996. Géographie électorale de la Gauche en Europe des années quatre-vingt et quatre-vingt-dix. In Lazar M. (ed.), La gauche en Europe depuis 1945. Invariants et mutations du socialisme européen, Paris, PUF.

Vandermotten C., Medina Lockhart P., 2000. Géographie électorale de l'Europe. In Perrineau P., Ysmal C. et al., Le vote des Quinze. Les élections européennes du 13 juin 1999, Paris, Presses de Sciences Po., p. 245-293.

Van Hamme G., 2008. La géographie de l'extrême-droite peut-elle s'expliquer par l'« ethnocentrisme » des territoires »? Une analyse multiscalaire en Europe occidentale. Espace, Populations, Sociétés, 3, p. 441-452.

Van Hamme G., 2012. Social classes and political behaviours: Directions for a geographical analysis. Geoforum, 43, 4, p. 772-783.

Vanlaer J., 1984. 200 millions de voix. Bruxelles, Société royale belge de géographie.

\section{NOTES}

1. De manière à offrir le panorama le plus large possible, cette cartographie est complétée par la prise en compte de la Norvège (à travers les résultats des élections législatives de 2013 et de 2009) et de la Suisse (élections de 2011 et de 2007). Quant à la Croatie, la première référence est celle des élections de 2011, qui ont désigné la première représentation de ce pays dans l'assemblée européenne.

2. Nous utiliserons régulièrement dans ce texte le terme "establishment " pour désigner l'ensemble des forces politiques proches des élites économiques, et régulièrement appelées à exercer le pouvoir politique dans un pays ou à y participer. Dans la suite de cet article, nous appliquerons ce terme aux forces politiques de gouvernement de centre-droit, mais aussi, avec des nuances, à la famille sociale-démocrate, en particulier dans ses composantes les plus réformistes, elle aussi fréquemment associée au pouvoir d'État et ayant souvent dès lors noué des liens organiques avec les élites d'importants secteurs de l'économie.

\section{ABSTRACTS}

The paper presents a synthetic cartography, at the regional scale, of the results of the 2014 European poll, with comparisons to 2009. It opens on a classification of the political parties, leading to an elementary typology : parties developing a leftist critics of the ruling forms of the European policy; parties agreeing with the ruling trends of the European agenda, either on a social-democratic or labour basis, or on a liberal, conservative or christian-democratic one ; eurosceptic or europhobic parties, on the basis of rightist and/or nationalistic attitudes. The 
paper examines also other variables, as abstention. Some regional conclusions appear, as for the political patterns in the main metropolitan areas, but the maps remain mainly a collection of national patterns. At the European scale, the explicit agreement to the ruling forms of the European construction gathers only a quarter of the potential voters, significantly less than in 2009. Moreover eurosceptic and europhobic Britain, the electoral disinterest for Europe is the strongest in the new Central-Eastern European member States.

L'article propose une cartographie synthétique, à l'échelle régionale, des résultats des élections européennes de 2014, avec des éléments de comparaison par rapport à 2009. Il s'ouvre sur une tentative de classification de l'ensemble des partis en lice, qui débouche sur une typologie simple : les partis s'inscrivant dans une logique de critique de gauche et/ou écologiste des formes dominantes de la construction européenne; les partis de tradition social-démocrate ou travailliste d'une part, libérale, démocrate-chrétienne ou conservatrice de l'autre, qui s'inscrivent dans des perspectives de continuité des formes dominantes de la construction européenne; les partis eurosceptiques, voire ceux refusant, sur une base de droite et de préférences nationales, les formes dominantes de cette même construction. L'article examine aussi d'autres variables, comme l'abstention. Au-delà de certaines récurrences régionales, par exemple concernant les comportements électoraux métropolitains, il montre que les cartes doivent avant tout être interprétées dans les contextes nationaux. A l'échelle européenne, l'adhésion explicite aux formes dominantes de la construction européenne ne rassemble que moins du quart de l'électorat potentiel, en sensible baisse par rapport à 2009. Outre la GrandeBretagne europhobe, le désintérêt électoral envers la construction européenne est particulièrement sensible dans les nouveaux États membres.

INDEX

Keywords: Europe, European poll, regional analysis, electoral geography

Mots-clés: Europe, élections européennes, analyse régionale, géographie électorale

\section{AUTHORS}

\section{CHRISTIAN VANDERMOTTEN}

Christian Vandermotten, cvdmotte@ulb.ac.be, Université Libre de Bruxelles, Académie Royale des Sciences, des Lettres et des Beaux-Arts de Belgique

\section{PABLO MEDINA LOCKHART}

Pablo Medina Lockart, Université Libre de Bruxelles 